\title{
Bone Marrow Transplant and Bangladesh Perspective
}

\author{
Abu Jafar Mohammed Saleh ${ }^{1}$ \\ ${ }^{1}$ Department of Hematology and Stem Cell Transplant, Evercare Hospital, Dhaka, Bangladesh.
}

\begin{abstract}
Citation: Saleh AJM. Bone Marrow Transplant and Bangladesh Perspective. Haematol J Bangladesh 2020; 4 (1):1-2
\end{abstract}

DOI: http: //doi.org/10.37545/haematoljbd 202053

*Correspondence: Abu Jafar Mohammed Saleh, Sr. Consultant, Hematology and Stem Cell Transplant, Evercare Hospital, Dhaka, Bangladesh. Contact: +880 1787 681748, email: ajmsaleh@gmail.com
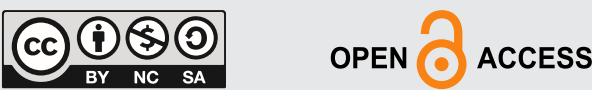

Copyright: (C) 2020 by author(s). This is an open access article published under the Creative Commons Attribution-Non Commercial-No Derivatives 4.0 International License, which permits use, distribution and reproduction in any medium or format, provided the original work is properly cited, is not changed in any way and is not used for commercial purposes. http:// creativecommons.org/licenses /by/4.0/
Bone marrow transplant is ideally known as stem cell transplant (SCT), is one of the curative options for many malignant and non-malignant haematological conditions. Since the first allogeneic SCT performed by E. Donnall Thomas in 1957, further development is ongoing to overcome the infectious and non-infectious complications. It took more than 50 years from the first published attempt to arrive at one million transplants by 2012 and currently more than 70,000 transplants are done annually in the world. Results are published in detail collecting data from available registries worldwide like Australasian Bone Marrow Transplant Recipient Registry (ABMTRR), African Blood and Marrow Transplantation Group (AFBMT), Asian Pacific Blood and Marrow Transplantation Group (APBMT), Bone Marrow Donors Worldwide (BMDW), Canadian Blood and Marrow Transplantation Group (CGBMT), the Centre for International Blood and Marrow Transplant Research (CIBMTR), European Society for Blood and Marrow Transplantation (EBMT), Eastern Mediterranean Blood and Marrow Transplantation Group (EMBMT), Latin American Blood and Marrow Transplantation Group (LABMT), and the World Marrow Donor Association (WMDA). ${ }^{1}$

Primarily SCT is two types - autologous and allogeneic. Autologous SCT is being used to salvage the patient after high dose chemotherapy and in allogeneic SCT, donor cells provide alloimmunity helps in graft-versus-tumour effect to eradicate residual disease and prevent relapse. Broadly autologous SCT is used in myeloma, lymphoma, and autoimmune diseases; on the other hand, allogeneic SCT is used in leukaemia, bone marrow failure and genetic diseases.

Availability of donors is the major concern for allogeneic SCT in which success mostly depends. Full matched HLA siblings are the most preferred donors but roughly it matches in around $30 \%$ cases. For the remaining majority cases we must depend on alternative sources like currently popular haplo-identical donors, cord blood or unrelated donors. Worldwide leading donor registries are: Australasian Bone Marrow Transplant Recipient Registry (ABMTRR), Centre for International Blood and Marrow Transplant Research (CIBMTR), the Austrian Registry (ASCTR), the Czech BMT Registry, the French Registry (SFGM), the German Registry (DRST), the Italian Registry (GITMO), the Dutch Registry
(HOVON), the Spanish BMT Registry (GETH), the Swiss Registry (SBST), the Turkish BMT Registry and the British Registry (BSBMT) and Eurocord ${ }^{1}$. To arrange a cord unit or unrelated donor from these registries is exceedingly difficult in terms of logistics and costly as well. To overcome this obstacle, we need to establish a national marrow registry through which many donors could be available.

Use of unrelated and haplo-identical donors, availability of more pre and post SCT chemo-immunotherapy, addition of newer drugs to treat graft-versus-host disease (GVHD), use of donor lymphocyte infusion (DLI) and improvement of supportive care all contributing significantly increasing the scope and outcome of SCT. Although SCT is a curative option but it does not give a guarantee of cure for all cases. The success rate of allogeneic SCT in marrow failure is more than $80 \%$ 
followed by hemoglobinopathies and in acute leukaemia, the relapse rate after allogeneic SCT is about 30 to 50 percent depending on conditioning regimen, GVHD prophylaxis and disease at which transplant is done. Besides relapse, nearly $30 \%$ suffers from long term complications like GVHD, hormonal and metabolic insufficiencies; infertility and impaired cognitive effect also contributes to long term or late complications. Moreover, huge cost and long term follow up remains a major issue.

A BMT centre should have infrastructure facilities, the optimization of blood transfusion services and radiology, and laboratory facilities for haematology, molecular biology, immunology, and the pharmacy facilities with access to chemotherapy and anti-infective agents. Availability of staff and their training and collaboration with national and international centres are also extremely important for newly developed centres.

In Bangladesh, SCT was started with autologous type at Dhaka medical college hospital in collaboration with the BMT team from Massachusetts general hospital, USA in March 2014. Subsequently SCT started at private level at Apollo hospitals (now Evercare hospital Dhaka) with autologous program in March 2016. First allogeneic SCT was done at Combined military hospital, Dhaka in April 2018 and currently these three hospitals are doing both autologous and allogeneic SCT routinely. Two other hospitals (BSMMU and Asgor Ali) did one autologous transplant each and under process of further development.

All these centres are facing certain challenges since beginning. Traditionally leukemic cases upon diagnosis mostly travels abroad to have their full treatment specially those who are rich; and some of them gets their chemotherapy part in Bangladesh but prefers the SCT part in abroad. This is the common scenario and it happens due to lack of confidence to our hospitals. This is the biggest challenge for the newly established centres to gain the confidence of our patients and have the full treatment locally. With our current population of more than 170 million, Bangladesh requires at least 5000 to 10000 SCT per year. ${ }^{1}$ The most important asking from patient side is local success rate and there is no straight cut answer at this time. We have started and it will take at least another five years to get the answer. The most responsibility depends on leading institutes both at government and private sectors. Success depends on physicians' experience, availability of laboratory and pharmacy support, and finally teamwork. Confidence does not develop in a day; the team who can handle the earlier part of treatment like induction, consolidation in a leukemic patient will do better in the transplant management as well. That is why it is a must to develop a good leukaemia service before starting transplant unit.

To retain the patients, we have two positive features like compliance with local environment and low cost. Certainly, the cost is much competitive and roughly it will be double in India, six times in Thailand and ten times more in Singapore. This estimation is done from those patients who were treated abroad and compared with local cost both at private and public hospitals in Dhaka.

Finally, accreditation by international bodies like the Foundation for the Accreditation of Cellular Therapy (FACT) or The Joint Accreditation Committee ISCT-Europe and EBMT (JACIE) will help in recognizing our centres globally and boost gaining confidence of our patients. Since then we must work hard to upgrade ourselves and gaining confidence.

\section{References}

1. Niederwieser D, Baldomero H, Szer J, Gratwohl M, Aljurf M, Atsuta Y, Bouzas LF, Confer D, Greinix H, Horowitz M, Iida M. Hematopoietic stem cell transplantation activity worldwide in 2012 and a SWOT analysis of the Worldwide Network for Blood and Marrow Transplantation Group including the global survey. Bone marrow transplantation. 2016 Jun;51(6):778-785. 